Ophthalmologe 2012 · 109:6-7

DOI 10.1007/s00347-011-2424-8

๑) Springer-Verlag 2012
C.H. Meyer

Klinik Pallas AG, Olten

\section{Smarte Ophthalmologen}

\section{"Smartphones for nothing and the Apps for free?"}

Jeden Monat kommen neue Apps auf den Markt, sodass man sich zunehmend die Frage stellt: Sind unter diesen Angeboten die medizinischen Apps effektiv und sind sie überhaupt sicher in der Patientenbetreuung $[1,2]$ ? Insgesamt betrachtet, spielen medizinische Apps bisher zwar nur eine untergeordnete Rolle, sie werden aber in den kommenden Jahren mit Sicherheit an Bedeutung zunehmen [3].

\section{\) Medizinische Apps werden in den kommenden Jahren an Bedeutung gewinnen}

Bisher entwickelte sich der App-Markt unkontrolliert und ohne Vorschriften dies obwohl eine Reihe medizinischer Apps, die insbesondere klinische Parameter bestimmen oder Daten erheben, wahrscheinlich unter das U.S. Food and Drug Administration (FDA) 510(k)-Gesetz für Medizinprodukte fallen würde [4-6]. Für das Frühjahr 2012 hat die FDA eine Überarbeitung ihrer Klassifikation für Medical Device Data Systems (MDDS) - sog. medizinische Datensysteme - angekündigt. Nach dieser Klassifikation werden Geräte, die zum Überwachen von Patienten eingesetzt werden, als Klasse II („special controll“) eingestuft und unterliegen damit einer Zulassung. Geräte, die nur Daten übermitteln, aber nicht kontrollieren oder verändern, werden dagegen als MDDS Klasse I („general control“) definiert. Um den geäußerten Sicherheitsbedenken zuvorzukommen, haben bereits einige App-Hersteller eine Zulassung bei der FDA für ihre medizinischen Apps be- antragt, da diese Apps möglicherweise auch unter das Medizinproduktegesetz fallen könnten.

In diesem Leitthema sollen in 2 Übersichtsarbeiten die wichtigsten medizinischen Apps für Augenärzte und für Patienten, hier insbesondere sehbehinderte Patienten, dargestellt werden. Die Breite und Fülle an angebotenen Apps ist unbeschreiblich groß und kann hier nur exemplarisch beleuchtet werden. Einige der Apps sollten sicher nicht als medizinische Apps verstanden werden und dienen eher zum Zeitvertrieb oder zur Unterhaltung. So kann man sich z. B. auf der Homepage des in die Jahre gekommenen Altrockers Lou Reed eine Lese-App mit größeren Buchstaben und Zahlen herunterladen, um bei gleichaltrigen Fans der zunehmenden Alterssichtigkeit zu begegnen. Andere Apps haben schon einen ernsten Hintergrund, spiegeln aber in ihren ersten Versionen noch die im medizinischen Wissen oft mangelnde Grundkenntnis der Programmierer wider. Dies ist auch der Grund, weshalb sich die in Anlehnung an den Dire-Staits-Titel „Money for nothin“ and chicks for free" gewählte Überschrift momentan noch als falsch herausstellt. Kostenlose Apps können hilfreich sein, besitzen aber bisher oft nicht die nötigen ausgereiften Programme. Auf der anderen Seite gibt es handwerklich sehr solide App-Programme wie das „Eye Handbook“, das mit Unterstützung der American Academy of Ophthalmology (AAO) konzipiert ist. Auch für sehbehinderte und blinde Patienten gibt es eine Reihe von sehr ausgereiften Programmen, die in enger Zusammenarbeit mit gehandicapten Patienten entstanden sind und von de- ren Dachorganisationen (UK's Royal National Institute of Blind People und dem American Federation for the Blind) überaus gelobt werden. Diese Plattformen bieten durch benutzerfreundliche Apps einen barrierefreien Zugang zum Internet und/oder zur Navigation im täglichen Leben. Natürlich verstehen sich viele dieser ersten App-Versionen noch als Prototypen, die noch nicht ausgereift und verbesserungswürdig erscheinen. Sie zeigen aber auch das große Potenzial von Smartphones und ihren App-Anwendungen im gegenwärtigen Alltag und in der modernen Medizin von morgen.

Es gibt natürlich auch Grenzen von Smartphones. So schlafen Assistenten im Bereitschaftsdienst unruhiger, wenn sie ein Smartphone direkt neben dem Kopfkissen liegen haben [7]. Daneben blieb auch die Tatsache weithin unbeachtet, dass das Telefon und heute insbesondere Smartphones ein beliebter Ort von Bakterien sind und demzufolge - wie einst die geliebte Krawatte an britischen Krankenhäusern - mit Vorsicht am Patientenbett zu nutzen sind und bestimmt nichts im Operationssaal zu suchen haben $[8,9]$.

Mit Steve Jobs ist vor wenigen Wochen der geistige Vater des Smartphones und Mitgründer von Apple verstorben. Sein Macintosh benutzte bereits 1984 als erster Computer keine Zeilencodes mehr, sondern eine grafische Benutzeroberfläche mit einer Computermaus für die Steuerbefehle. Später hat er Design-Ikonen geschaffen, mit dem tragbaren $\mathrm{Mu}$ sikabspielgerät iPod die Musikindustrie umgekrempelt und Apple zum Kultkonzern gemacht. Mit dem iPhone revolutionierte er den Handy-Markt und schuf mit 


\section{- \\ dem iPad einen neuen Markt für „Digital Lifestyle-Produkte“ [10]. Steve Jobs' Ver- mächtnis hat und wird unsere reale und digitale Welt nachhaltig verändern. \\ Ohne Anspruch auf Vollständig- keit oder letzte Aktualität versucht die- ses Leitthemenheft die unterschiedlichen Aspekte der medizinischen Apps für Ärz- te und Patienten in einer kritischen Dar- stellung zu beleuchten. Eine aktuelle Lis- te interessanter medizinischer Apps findet der interessierte Leser auf der Homepage www.klinik-pallas.ch. \\ Korrespondenzadresse

Prof. Dr. C.H. Meyer
Klinik Pallas AG
Louis Giroud-Str. 20,
4600 Olten, Schweiz
meyer_eye@yahoo.com

\section{Literatur}

1. Kwok R, Terry M (2009) Personal technology: Phoning in data. Nature 458:959-961

2. Stone R (2010) Dialing up knowledge - and harvests. Science 327:808

3. Gross H (2011) Smartphones - how useful are medical Apps? Dtsch Med Wochenschr 136:17

4. Reichertz PS (1997) Understanding government regulation of the marketing and advertising of medical devices, drugs, and biologics: the challenges of the Internet. Food Drug Law J 52:303-308

5. Chaps NA (2010) Pharmaceuticals and medical devices: FDA oversight. End-of-year issue brief. Issue Brief Health Policy Track Serv 4:1-31

6. Kesselheim AS (2010) Permitting product liability litigation for FDA-approved drugs and devices promotes patient safety. Clin Pharmacol Ther 87:645647

7. Danker-Hopfe H, Dorn H, Bornkessel C, Sauter C (2010) Do mobile phone base stations affect sleep of residents? Results from an experimental double-blind sham-controlled field study. Am J Hum Biol 22:613-618

8. Saxena S, Singh T, Agarwal H et al (2011) Bacterial colonization of rings and cell phones carried by health-care providers: are these mobile bacterial zoo in the hospital? Trop Doct 41:116-118

9. Saver C (2011) Cell phones are everywhere, but do they belong in the OR? OR Manager 27:13-14

10. Wong D, Joussen A (2011) Welcome to the iPad generation. Graefes Arch Clin Exp Ophthalmol 249:1-2

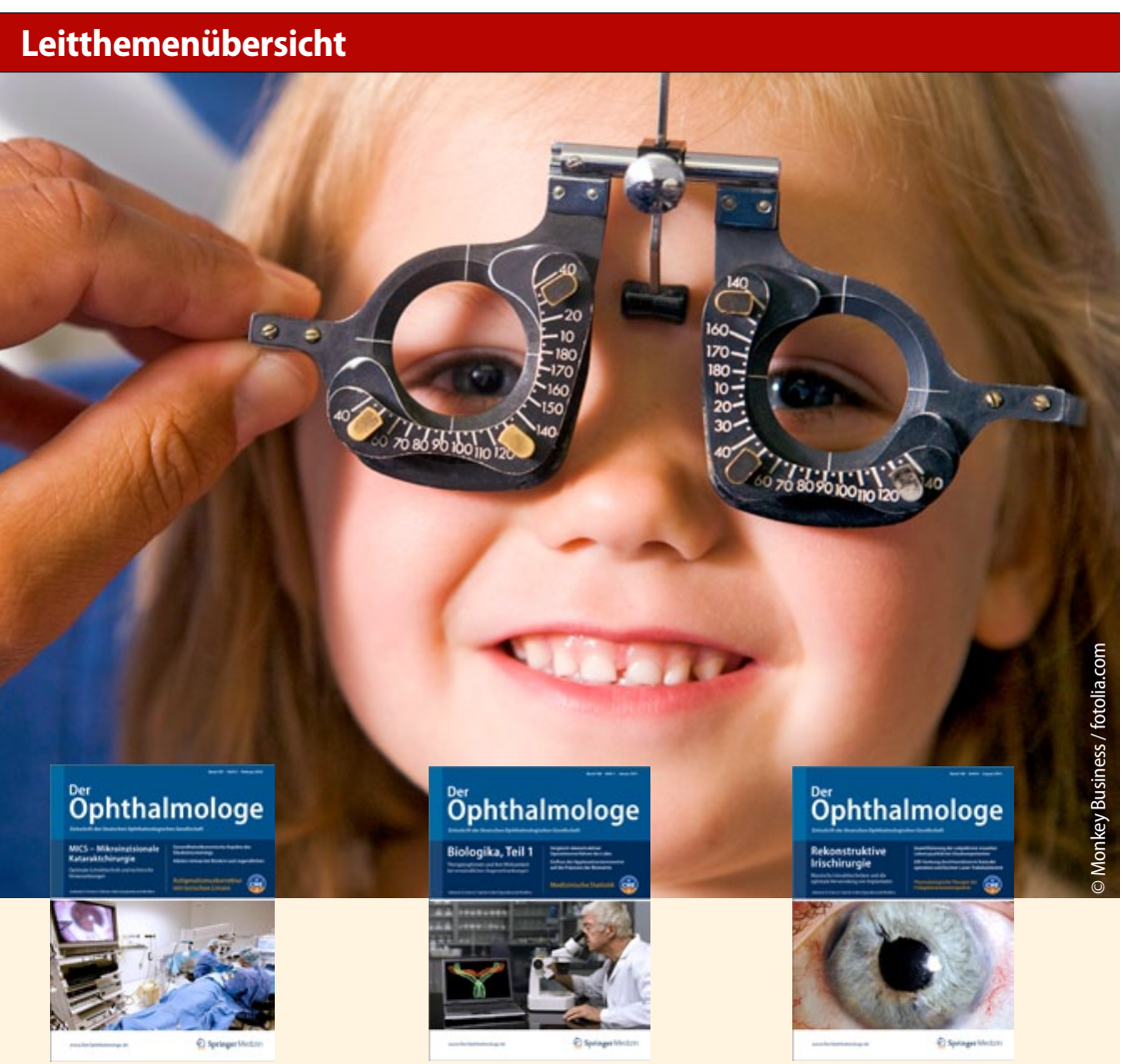

Der Ophthalmologe bietet Ihnen jeden Monat umfassende und aktuelle Beiträge zu interessanten Themenschwerpunkten aus allen Bereichen der Ophthalmologie.

Möchten Sie ein bereits erschienenes Heft nachbestellen? Einzelne Ausgaben können Sie direkt bei unserem Kundenservice zum Preis von je EUR 35,- zzgl. Versandkosten beziehen:

\section{ÜBERBLICK}

2011

01/11 Biologika, Teil 1

02/11 Venöse retinale Gefäßverschlüsse

03/11 Biologika, Teil 2

04/11 Fallstricke der Brillenbestimmung

05/11 Orbitaerkrankungen, Teil 1

06/11 Orbitaerkrankungen, Teil 2

07/11 Glaukom im Kindesalter

08/11 Rekonstruktive Irischirurgie

09/11 Perspektiven der Hornhauttransplantation

10/11 Verätzungen und Verbrennungen des Auges

11/11 Okuläre Hypertension

12/11 Augenschmerzen aus interdisziplinärer Sicht

So erreichen Sie unseren Kundenservice:

Springer Customer Service Center GmbH

Kundenservice Zeitschriften

Haberstr. 7

69126 Heidelberg

Tel.:+49 $6221345-4303$

Fax:+49 $6221345-4229$

E-Mail:leserservice@springer.com

www.DerOphthalmologe.de

\section{VORSCHAU}

2012

01/12 Smartphones in der Augenheilkunde

02/12 Therapieansätze bei erblichen Netzhautdystrophien

03/12 Ophthalmologische Therapie in der Schwangerschaft

04/12 Frühdiagnostik und Therapie suprasellärer Tumoren

05/12 Funktionelle Glaukomdiagnostik Alternativen zum Gesichtsfeld

06/12 Morbus Behcet 